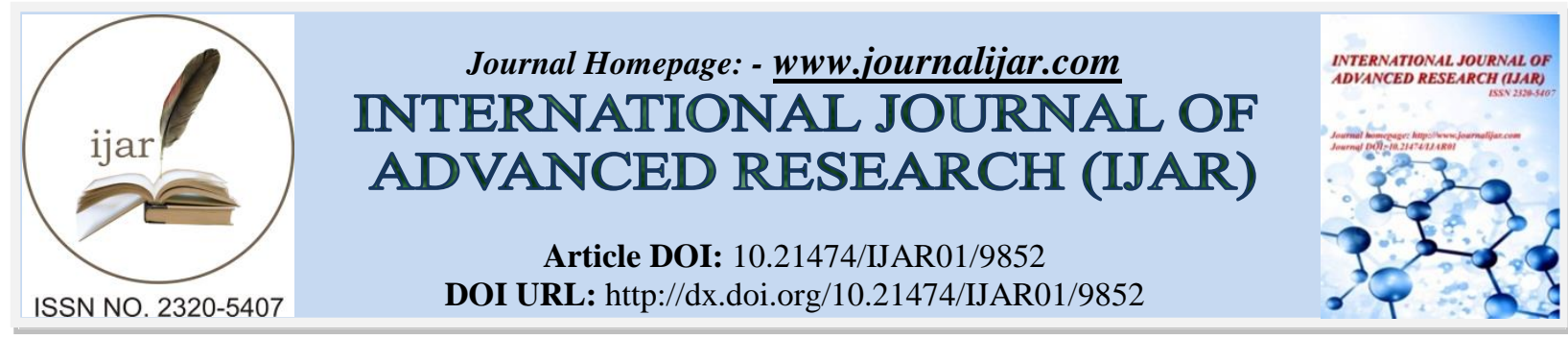

RESEARCH ARTICLE

\title{
EVALUATION DE L'EFFICACITE DES PIEGES LOCAUX DES MOUCHES DES FRUITS (DIPTERA : TEPHRITIDAE) DANS LES ZONES DE FAIBLES PRODUCTIONS FRUITIERES AU CENTRE-OUEST (DALOA ; COTE D'IVOIRE).
}

\author{
N'Dépo O. $R^{1}$, Yeboué N. L ${ }^{1}$. Achiépo G. $M^{1}$, Minhibo Y. $M^{2}$ and Coulibaly $A^{1,2}$.
}

1. Univertisté Jean Lorougnon Guédé (Daloa), Laboratoire d'Amélioration de la Production Agricole, BP 150 Daloa (Côte d'Ivoire).

2. Centre National de Recherche Agronomique (CNRA), 01 BP 1740 Abidjan 01, (Côte d'Ivoire).

\section{Manuscript Info}

…......................

Manuscript History

Received: 08 August 2019

Final Accepted: 10 September 2019

Published: October 2019

Key words:-

Côte d'Ivoire / Daloa / fruit flies / Local traps / synthetic traps.

\section{Abstract}

West African countries have been dealing with the problem of fruit flies for many years. Côte d'Ivoire is particularly affected by this problem due to the export mangoes' production. Detecting the presence of flies in a locality requires the use of synthetic traps that are inaccessible to Ivorian producers. The objective of this study is to evaluate the effectiveness of local for the capture of fruit flies. To this end, local and synthetic traps have been installed at the Daloa University site and the collect of captured flies have been carried out each month. A total of 76593 fruit flies were captured and divided into eleven species. Bactrocera dorsalis is the most abundant with $92.91 \%$ and a daily catch of 14.16 individuals / day / trap. Mean capture of local traps (25.09 individuals) and synthetic traps (43.09 individuals) are statistically identical. Population growth peaks coincide with the rainy season (May and June). Local traps can be used in the detection and monitoring of fruit flies.

Copy Right, IJAR, 2019,. All rights reserved.

\section{Introduction:-}

La production fruitière et horticole contribue au développement de l'Afrique, plus particulièrement l'Afrique de l'Ouest. Ainsi d'importantes quantités de produits horticoles et fruitiers dont la mangue sont-elles exportées vers les marchés européens. Ces fruits dont ces fruits jouent un rôle majeur dans le marché local, national, régional et international (Vayssières et al., 2004). En Côte d'Ivoire, la production nationale de mangues exports est estimée à environ 180000 tonnes. Cependant, seulement 32400 tonnes / an de cette production est exportée vers le marché européen à cause des dégâts des mouches fruits estimés à plus de 40\% (Anonymes, 2018). En effet, les fruits et les légumes sont continuellement menacés par des insectes nuisibles. Parmi ceux-ci, figurent les mouches des fruits, dont 250 espèces sont économiquement nuisibles au plan mondial (White \& Elson-Harris, 1992). En Côte d'Ivoire, les mangues sont fortement attaquées par l'espèce Batrocera dorsalis N'Dépo et al. (2009; 2010). Les pertes imputées à cette espèce sont estimées entre $17 \%$ et $80 \%$ durant la saison des mangues, voire plus (N'Dépo, 2010). Face à cette nuisance économique des mouches des fruits, la localité de Daloa, faible zone de production fruitière, n'est pas en marge de l'invasion de ces mouches du fait de l'agriculture péri-urbaine qui s'y pratique. En effet, les fruits piqués par les mouches hébergent des œufs et des larves. Ces fruits passent souvent inaperçus au contrôle phytosanitaire ou sont parfois délaissés par ignorance dans les vergers. Ainsi, constituent-ils des foyers d'infestation pour la zone. En revanche, la détection de la présence des mouches dans une localité exige l'utilisation des pièges 
synthétiques spécifiques aux Tephritidae. Malheureusement, ces pièges et leurs composants sont difficiles d'accès en Afrique et en Côte d'Ivoire en particulier. Toutefois, la possibilité de réduire les dégâts des mouches en zones de production de mangues, nécessite un système d'alerte d'où l'utilisation des pièges locaux (mesures préventives) sont envisagés afin de pouvoir donner l'alerte au moment opportun. Cette étude a pour objectif d'évaluer l'efficacité des pièges locaux des mouches des fruits sur le site de l'Université Jean Lorougnon Guédé de Daloa.

\section{Matériel et methodes:- Milieu d'étude}

Les travaux se sont déroulés de Décembre 2017 à Septembre 2018 à l'Université Jean Lorougnon Guédé (UJLoG) de Daloa. Daloa est située au Centre-Ouest de la Côte d'Ivoire avec pour coordonnées géographiques 6²6'17' de longitudes Ouest et $6^{\circ} 54^{\prime} 40^{\prime \prime}$ de latitudes Nord. La région est caractérisée par une flore très variée avec deux types de végétations bien distinctes: une zone forestière et une zone de savane. On y rencontre également des cultures de rente telles que le cacao, le café, le palmier à huile, l'anacarde et l'hévéa et des essences forestières. Le climat est de type équatorial et subéquatorial deux grandes saisons. La saison part d'Avril à mi-Octobre et la saison sèche de miNovembre à mi- Mars. La pluviométrie annuelle est passée de 1868,5 mm en 1968 à $1200 \mathrm{~mm}$ en 2014 Ligban et al. (2009). La température moyenne oscille entre $22,01^{\circ} \mathrm{C}$ à $25,23^{\circ} \mathrm{C}$ (N'Guessan et al., 2014).

\section{Matériel et méthodes}

\section{Piégeage des mouches des fruits}

Les attractifs sexuels (Méthyl Eugénol, Terpinyl Acétate, Cue Lure et Trimedlure) ont été ôtés de leur emballage et placés dans la nacelle située sous le couvercle et l'insecticide solide (diclorvos) au fond du piège. Le réseau de piégeage et les relevés hebdomadaires des pièges ont été exécutés suivant la méthodologie standard (Vayssières \& Sinzogan, 2008). Les données climatiques (Température et Pluviométrie) ont été également enregistrées. La prévalence des mouches (FTD) a été également déterminée. Au total 8 pièges synthétiques et 8 pièges locaux (boîte de Nesquik) ont été installés de manière aléatoire, soit seize (16) pièges sur l'ensemble du site. L'essai a été répété 2 fois.

\section{Critères de reconnaissance et identification des espèces capturées}

Les Tephritidae ont été identifiées à l'aide d'une loupe binoculaire de marque «LEICA EZ4 » et de guides d'identification des mouches (De Meyer, 2000, Ekesi \& Billah, 2007). Ces guides renferment des images et des traits caractéristiques de reconnaissance des mouches permettant d'attribuer un nom scientifique aux mouches des fruits.

\section{Détermination de la prévalence des différentes espèces de mouches}

Les informations portant sur les effectifs des différentes espèces de mouches ont permis d'estimer leur niveau de prévalence en calculant le nombre moyen de mouches dans un piège en une journée (FTD) par la formulation suivante (Amevoin et al., 2009 ; CIPV, 2016) :

$$
\text { FTD }=\quad \begin{array}{ll}
\mathrm{F} & \begin{array}{l}
\mathrm{F}=\text { nombre total de mouches capturées /espèce } \\
\mathrm{T}=\text { nombre de pièges inspectés } \\
\mathrm{D}=\text { nombre de jours d'exposition des pièges sur le terrain }
\end{array}
\end{array}
$$

\section{Exploitation statistique des données}

L'ensemble des données collectées a été soumis aux traitements statistiques au moyen du logiciel STATISTICA. v 7.1. L'abondance des mouches par type de piège, par attractif et dans l'ensemble, ont été soumises à une analyse de variance (ANOVA) au seuil de 5\%. Les moyennes obtenues sont classées suivant le test de Student Newman Keuls. Les données des pièges locaux et synthétiques ont été comparées par le Test t de Fisher.

\section{Résultats et discussion:- \\ Résultats}

Abondance relative des mouches capturées avec les pièges locaux

Les pièges locaux ont permis de capturer 28106 Tephritidae adultes réparties en trois (3) genres et huit (8) espèces. Il s'agit notamment de Bactrocera dorsalis, Zeugodacus cucurbitae, Dacus punctatifrons, Dacus theophratus, Dacus bivittatus, Dacus diastatus, Ceratitis capitata, et Ceratitis bremii. L'abondance relative des mouches varie 
d'une espèce à une autre. Elle est fortement exprimée chez B. dorsalis avec 92,14\%. Les autres espèces faiblement capturées, présente une abondance relative fluctue entre 0,004 et $4,42 \%$ (Figure 1).

\begin{abstract}
Abondance relative des mouches capturées avec les pièges synthétiques
48487 individus ont été capturés par les pièges synthétiques et réparties en t neuf (9) espèces. Il s'agit de Dacus longistylus (espèce nouvelle en Côte d'Ivoire), Dacus humeralis, Ceratitis cosyra et les espèces capturées par pièges locaux à l'exception de $D$. diastatus et $C$. bremii. $B$. dorsalis demeure également majoritaire avec $92,36 \%$. Les autres espèces présentent une abondance relative compris entre 0,002 et 3,46\% (Figure 2). Dans l'ensemble, $B$. dorsalis est l'espèce dominante avec un taux de $92,28 \%$.
\end{abstract}

\title{
Captures moyennes des mouches par attractifs et leur prévalence
}

La capture moyenne des mouches des fruits par semaine et par attractifs varie d'un attractif à un autre et d'un piège à un autre. Le méthyl eugénol présente le taux de capture le plus élevé $(92,91 \%)$ sur l'ensemble des attractifs. La capture moyenne du méthyl eugénol est estimée à 69,52 $\pm 20,90$ individus avec les pièges locaux et 100,98 $\pm 23,80$ individus avec les pièges synthétiques. Cette capture est évaluée à 18,82 $\pm 7,12$ individus pour le terpinyl Acétate, $9,25 \pm 2,19$ individus pour le Cue lure et 2,78 $\pm 0,97$ individus pour le trimedlure au niveau des pièges locaux (Figure 3). Au niveau des pièges synthétiques, elle est de 23,44 $\pm 8,35$ individus pour le terpinyl Acétate, 10,25 \pm 1,85 individus pour le cue lure et $0,02 \pm 0,02$ individus pour le trimedlure (Figure 4). Quant à la prévalence des mouches sur le site, elle est estimée à 14,61 individus/jr/piège pour B. dorsalis (espèce majoritaire) et fluctue entre 0,0008 et 2,41 individus/jr/piège pour les autres espèces. Parmi les attractifs sexuels, le méthyl Eugénol s'est révélé le plus efficace.
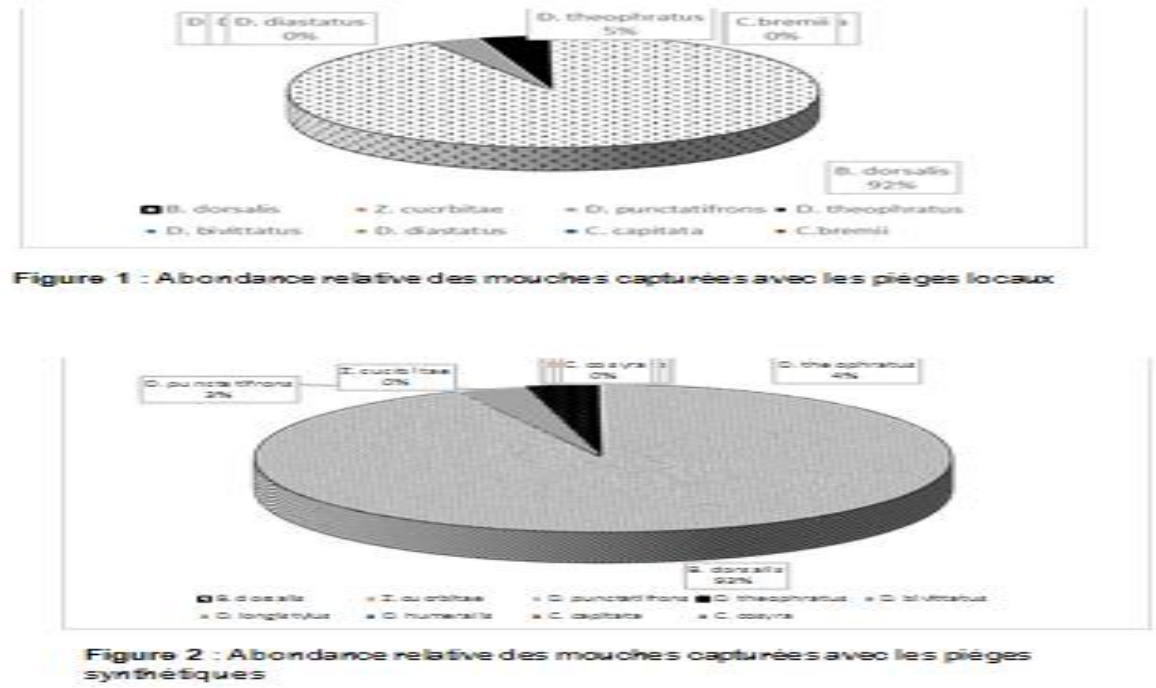


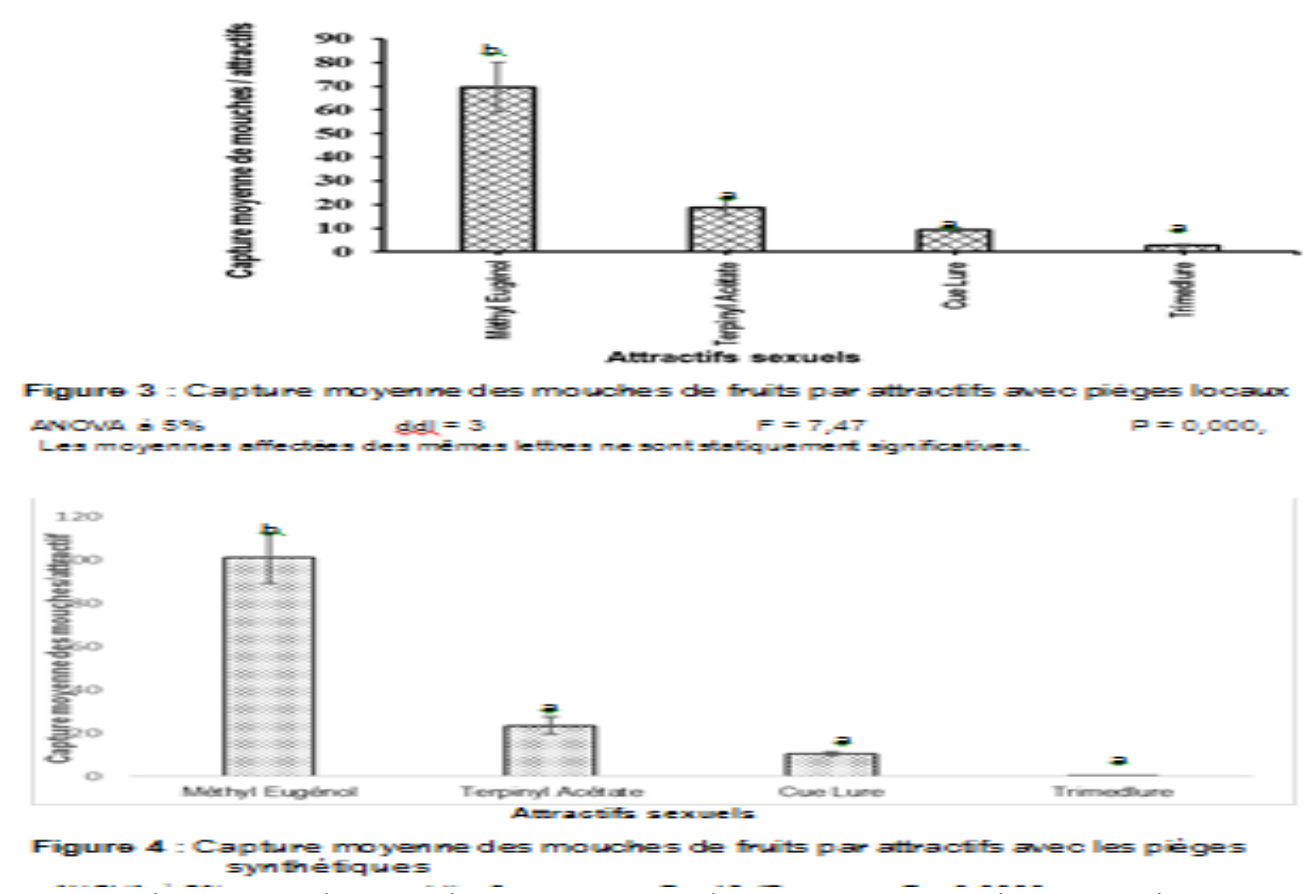

Etude comparée des données de piégeàge entre les pièges locaux et les pièges synthétiques

La capture moyenne de mouches varie entre les deux types de pièges. Elle est estimée à 43,09 $\pm 8,17$ individus pour les pièges synthétiques et à 25,09 $\pm 5,60$ individus pour les pièges locaux. Le test $t$ de Fisher ne révèle de différence significative entre la capture moyenne des pièges $(\mathrm{P}=0,07)$ (Figure 5).

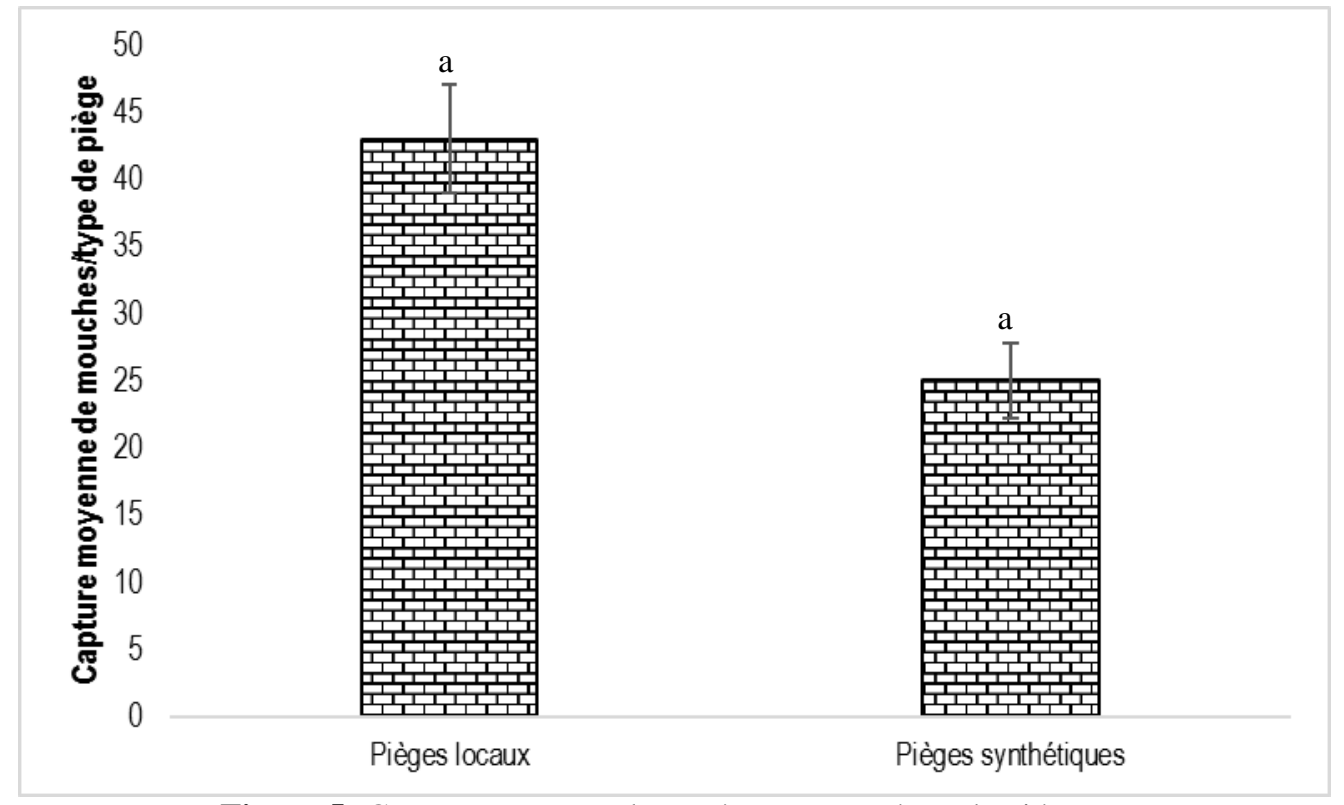

Figure 5:-Capture moyenne des espèces par système de piégeage

\section{Dynamique des populations des mouches des fruits}

Cette étude est axée sur les espèces présentes durant toute la période du piégeage. Il s'agit en occurrence de $B$. dorsalis et du genre Dacus. Partir de 892 individus en Décembre 2017, la population de B. dorsalis a baissé brusquement jusqu'à atteindre 386 mouches en Février 2018. Puis la population augmente rapidement jusqu'atteindre un pic de croissance (26 779 mouches) en Mai 2018. Ensuite, la population diminuer progressivement jusqu'au mois de Septembre 2018 (48 mouches). Quant au genre Dacus, la population croît 
faiblement à partir Mai 2018 pour atteindre un pic (2 416 individus) en Août 2018. Puis, elle baisse progressivement en Septembre 2018. Il convient de note que tous les pics de croissances des mouches sont observés pendant la saison des pluies (Figure 6).

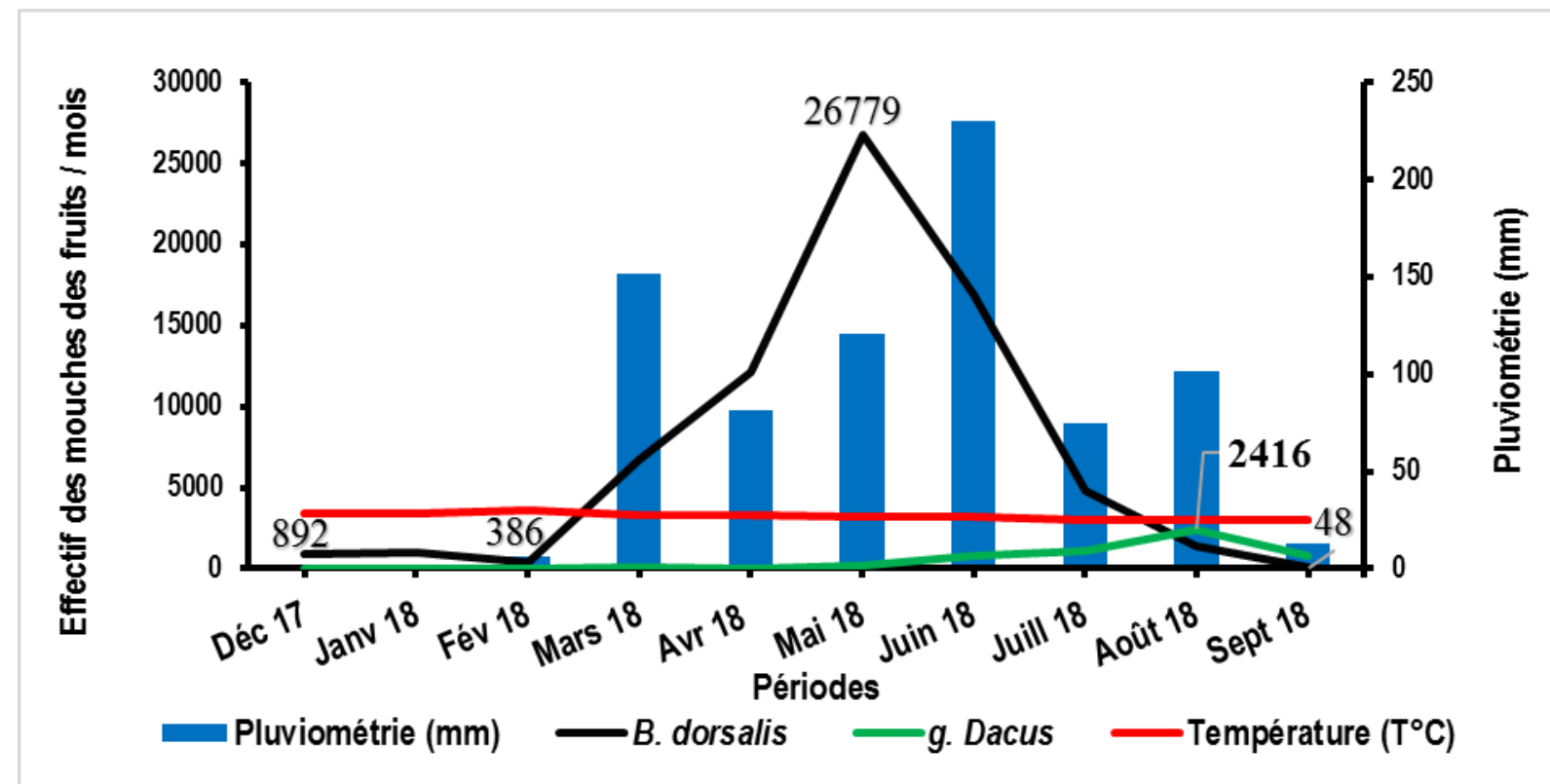

Figure 6:-Fluctuation spatio-temporelle de la population de B. dorsalis et du genre Dacus sur le site de UJLoG de Décembre 2017 à Septembre 2018

\section{Discussion:-}

Au total, 11 espèces des mouches des fruits ont été capturées sur l'ensemble du système de piégeage à Daloa. La plupart de ces espèces avaient été déjà identifiées en Côte d'Ivoire (Hala, 2001 ; Ouattara, 1998) et dans d'autres régions d'Afrique (Mwatawala et al., 2004 ; Vayssières et al., 2004 et 2005). B. dorsalis a été l'espèce majoritaire sur le site. Cette impressionnante présence s'expliquerait par une forte invasion de la zone Daloa par cette espèce de Tephritidae. En effet, la ville de Daloa, de par sa situation géographique, reçoit une importante quantité de fruits dont les mangues d'exportation, un hôte préféré de B. dorsalis, en provenance des zones productrice de manges. Mwatawala et al. (2009); Ekesi et al. (2009), spéculent que la mangue est l'un des fruits préférés de B. dorsalis et par conséquent, elle pullule dans les zones productrices de mangues. La faible capture des espèces indigènes serait due au statut de super compétiteur de $B$. dorsalis vis-à-vis de ces espèces mais également du fait de l'existence d'une compétition interspécifique entre les espèces (Duyck et al., 2004). La présence de quelques plants fruitiers sur le site d'une part et la convergence de mangues des régions infestées vers la localité à Daloa d'autre part, justifieraient également l'abondance de cette mouche dans ladite localité. Le suivi de la dynamique des populations de mouches des fruits, révèle des pics de croissance de B. dorsalis et du genre Dacus en saison des mangues qui coïncident avec la saison des pluies. Ainsi, le maximum de capture de mouches est-il observé au cours de de la saison pluvieuse. Cette abondance pourrait être due à une synchronisation du cycle biologique de la mouche avec le stade phénologique de la mangue coïncidant avec la saison des pluies. Ainsi, se produirait-il une importante émergence de mouches adultes du sol à cette période de l'année (Duyck et al. 2007 ; Vayssières et al., 2009). Ces mêmes auteurs avaient révélé à cet effet, une corrélation positive entre les facteurs climatiques (la température, l'humidité relative et la pluviométrie) et la fluctuation de la population de B. dorsalis et les autres espèces. Aussi face à la grande capacité d'adaptation des mouches, N'Dépo (2010) souligne que ces dernières sont capables de s'accommoder facilement aux conditions environnementales d'une zone donnée pour s'y développer convenablement. Au cours du piégeage, le méthyl eugénol a capturé plus de mouches que les autres attractifs. Ce constat expliquerait par le fait que cet attractif à un large spectre d'action et est spécifique des mâles du genre Bactrocera et du sous genre Ceratitis (White \& Elson-Harris, 1992). Une importante capture de B. dorsalis a été observée par Coulibaly (2017) et Minhibo et al. (2018), lors de travaux sur l'efficacité du système de piégeage des mouches des fruits au nord de la Côte d'Ivoire. Ces résultats sont en accord avec ceux de Vayssières et al. (2008) au Bénin, Mwatawala et al. (2009) en Tanzanie et N'Dépo et al. (2013) en Côte d'Ivoire. Selon Minhibo et al. (2018), le méthyl eugénol est un puissant attractif chimique qui peut diffuser environ sur près de $2,5 \mathrm{~km}$ si bien que 
les mouches du genre Bactrocera sont fortement attirés. Au niveau des différents systèmes de piégeage, les espèces capturées par les pièges locaux étaient pour la plupart identiques à celles capturées par les pièges synthétiques. L'importante capture des pièges locaux s'expliquerait par la couleur jaune des bocaux qui est très attirante pour les insectes en général, en plus des attractifs qu'ils contiennent. De même, Mille (2010), souligne que la forme sphérique et la couleur jaune des pièges sont des facteurs attractifs des insectes en particulier.

\section{Conclusion:-}

Onze espèces de mouches des fruits ont été identifiées à Daloa. B. dorsalis est l'espèce majoritaire.

Sa capture journalière est estimée à 14,92 individus/jour/piège. Les espèces indigènes sont faiblement représentées. Le pic de croissance des mouches coïncide largement avec la saison des mangues et des pluies. Les pièges locaux sont efficaces dans le piégeage et suivi du monitoring des mouches des fruits.

\section{Références :-}

1. Amevoin K., Sanbena B. B., Nuto Y., Gomina M., De Meyer M. \& Glitho I. A. (2009). Les mouches des fruits (Diptera : Tephritidae) au Togo : inventaire, prévalence et dynamique des populations dans la zone urbaine de Lomé. Int. J. Biol. Chem. Sci 3(5) : 912-920.

2. Anonymes (2018). Filière mangue. 180000 tonnes par an, $3^{\text {ème }}$ fournisseur sur le marché Européen. Résultats et impacts. Lettre d'information N7, CICG. www.gouv.ci/impact-développement/ , Consulté le 13/10/2019.

3. CIPV (2016). Établissement de zones exemptes de mouches des fruits (Tephritidae). Normes Internationales pour les Mesures hytosanitaires, NIMP 26, FAO, 64 p.

4. Coulibaly. A. (2015). Evaluation des différents systèmes de piégeages des mouches des fruits. Mémoire de Master 2, Université Jean Lorougnon Guédé, 47p.

5. De Meyer M. (2000). Systematic revision of the subgenus Ceratitis Macleay s.s. (Diptera: Tephritidae). Zoological journal of Linnean Society, 128: 439-467.

6. Duyck P. F., David P. \& Quilici S. (2004). A review of relationships between interspecific competition and invasions in fruit flies (Diptera : Tephritidae). Ecological Entomology 29 : 511-520.

7. Duyck P. F., David P. \& Quilici S. (2007). Can more K-selected species be better invader? A case study of fruit fies in the Réunion. Diversity and distribitions (Diversity Distrib.), 13: 535-543.

8. Ekesi S. \& Billah M.K. (2007). A field guide to the management of economically important Tephritidae Fruit flies in Africa; second Edition, 55p.

9. Ekesi S., Billah M.K., Nderitu P.W., Lux A.S. \& Ivan R. (2009). Evidence for competitive displacement of Ceratitis cosyra by the invasive fruit fly Bactrocera invadens (Diptera: Tephritidae) on mango and mechanisms contribution to the displacement. Journal of entomology, 102(3): 98-991.

10. Hala N.F. (2001). Lutte contre les mouches des fruits, conv. CNRA/ICIPE, Rapport Exécution Technique 2000, Document Interne, CNRA, Abidjan, Côte d'Ivoire, $11 \mathrm{p}$.

11. Ligban R., Gone L.D., Kamagate B., Saley M.B. \& Biemi J. (2009). Processus hydrogéochimique et origine des sources naturelles dans le degré carré de Daloa, $17 \mathrm{p}$.

12. Mille C. (2010). Les mouches des fruits de Nouvelle-Calédonie : (Diptera : Tephritidae). -

13. Systématique, comportement, dynamique et gestion des populations. Thèse de Doctorat, Université de la Nouvelle-Calédonie, Nouvelle-Calédonie, 349p.

14. Minhibo M.Y., N’Dépo O R., Hala N., Koua H., Tuo Y., N’Goran A., Coulibaly A., Doumbia S., Djaha J-B.A., Kouakou K.C., N’Da A.A., N'Da H.A \& Djidji H. (2018). Assessment of Fruit Fly Trapping System in Mango Orchards in Northern Côte d'Ivoire. J. Agri. Sci. Tech A8 18-27

15. Mwatawala M.W., White I.M., Maerere P.A., Sekondo F.J., \& De Meyer M. (2004). A new invasive Bactrocera species (Diptera: Tephritidae) in Tanzania. African Entomology, 12 (1): 154-156.

16. Mwatawala M.W., De Meyer M., Makundi R.H. \& Maerere A.P. (2009). An Overview of Bactrocera (Diptera: Tephritidae) Invasions and their Speculated Dominancy over Native Fruit Fly Species in Tanzania. Journal of Entomology, 6(1):18- 27, 10p.

17. N'Dépo O.R. (2010). Biologie et écologie de Bactrocera invadens Drew, Tsuruta et White, 2005 (Diptera : Tephritidae), principal déprédateur des vergers fruitiers en Côte-d'Ivoire : possibilité de lutte chimique raisonnée contre les mouches des fruits. Mémoire de Thèse Unique de l'Université de Cocody, (Abidjan, Côte d'Ivoire), $177 \mathrm{p}$.

18. N’Dépo O.R., Hala N.F., Allou K., Aboua L.R., Kouassi K.P., Vayssières J.F. \& De Meyer M. (2009). Abondance des mouches des fruits dans les zones de production fruitière de Côte d'Ivoire : dynamique des populations de Bactrocera invadens (Diptera : Tephritidae). Fruits, 64 (5): 313-324. 
19. N'Dépo O. R., Hala N.F., Gnago A., Allou K., Kouassi K. P., Vayssières J.F. \& De Meyer M. (2010). Inventaire des mouches des fruits de trois régions Agro-écologiques et plantes-hôtes associées à l'espèce nouvelle Bactrocera invadens Drew (Diptera : Tephritidae) en Côte d'Ivoire. European Journal of Scientific Research, 46 (1) : 63-72.

20. N'Dépo O. R., Hala N., N'Da A. A., Coulibaly F., Kouassi K. P., Vayssieres J. F. \& De Meyer M. (2013). Fruit flies (Diptera: Tephritidae) populations Dynamic in mangoes production zone of Côte d'Ivoire. Agricultural Science Research Journal 3 (11); pp. 352- 363.

21. N'Guessan A. H., N'Guessan K. F., Kouassi K. P., Kouamé N. N. \& N'Guessan P. W. (2014). Dynamique des populations du foreur des tiges du cacaoyer, Eulophonotus myrmeleon. Felder (Lépidoptère : Cossidae) dans la région du Haut-Sassandra en Côte d'Ivoire, 9 p.

22. Ouattara M. (1998). Fluctuation des populations des mouches des fruits (Diptera: Tephritidae) dans la région de Korhogo. Mémoire de DAA, INP/ESA, Côte d'Ivoire, 46p.

23. Vayssières J.F., Fatogoma S. \& Moussa N. (2004). Inventaire des espèces de mouches des fruits (Diptera: Tephritidae) inféodées au manguier au Mali et essai de lutte raisonnée. Fruits, 59(1): 1-14.

24. Vayssières J.F., Goergen G. Lokossou O. Dossa P. \& Akponon C. (2005). A new Bactrocera species in Benin among mango fruit fly (Diptera: Tephritidae) species. Fruits, 60: 371-377.

25. Vayssières J.F. \& Sinzogan A. (2008). Piégeage de détection des mouches des fruits. Projet régional de lutte contre les mouches des fruits en Afrique de l'Ouest. Fiche $N^{\circ} 3$, IITA-CIRAD, 4p.

26. Vayssières J.F., Korie S. Coulibaly O. Melle C.V. Temple L. \& Arinloye D. 2009. The mango tree in central and northern Benin: damage caused by fruit flies (Diptera: Tephritidae) and computation of economic injury level. Fruits, (64) : 207-220.

27. White I.M \& Elson-Harris M. (1992). Fruit flies of economic significance: their identification and bionomics. Cab Int., Aciar, Redwood Press, Melksham, UK, 601p. 